\title{
REVISIÓN Y ANÁLISIS DE ESTUDIOS DE MODELOS DE CAMBIOS DE USOS DEL SUELO Y DE ESCENARIOS A FUTURO
}

\author{
Marta Gallardo \\ Departamento de Geografía, Universidad de Murcia, \\ Calle Santo Cristo 1, 30001 Murcia \\ marta.gallardo@um.es
}

\begin{abstract}
Resumen: En las últimas décadas se ha producido un gran desarrollo de técnicas y modelos para la captura y el análisis de dinámicas de usos del suelo. Dicho interés se demuestra en los diversos programas institucionales y las numerosas investigaciones que se han llevado a cabo con el objeto principal de valorar y abordar un desarrollo sostenible del territorio. La sostenibilidad tiene implicaciones muy diversas, por lo que dichos estudios son también muy variados dependiendo del objetivo que se pretende alcanzar. Los modelos se han hecho cada vez más complejos, desde simples extrapolaciones a modelos estadísticos-matemáticos que compilan varias técnicas. Se presenta una revisión bibliográfica de trabajos sobre modelos de cambios de usos del suelo y de escenarios a futuro. Se definen las grandes tipologías de modelos que existen, las metodologías, factores explicativos, temáticas y modelos empleados, internacionalmente y en España. Se presentan también los grandes problemas y retos que afronta esta materia.
\end{abstract}

Palabras clave: cambios de usos del suelo, escenarios a futuro, modelos, análisis teórico-bibliográfico, revisión.

\begin{abstract}
In the last decades there has been a broad development of techniques and models to capture and analyze land use dynamics, at global, regional, national and local scales. This interest is shown in the several institutional programs and studies that have been carried out with the main purpose of assessing and addressing a sustainable development of the territory. Sustainability has very different implications, so these studies are also
\end{abstract}

Recibido: 05-06-2018. Aceptado: 12-11-18. 
wide-ranging depending on their objective. Models have become more complex, from simple extrapolations to statistical-mathematical models that compile different techniques. A review of different land use change and future scenarios works is presented, describing different typologies, methodologies, driving factors and models used, worldwide and in Spain. Finally, the main problems and challenges faced by this subject are presented.

Keywords: land use change, future scenarios, models, theoretical analysis, review.

\section{Introducción}

El cambio de uso del suelo está causado por múltiples factores que interactúan entre sí. El conjunto de las fuerzas que provocan dichos cambios varía en el tiempo y en el espacio, dependiendo de las condiciones específicas humanas y ambientales que puedan darse. La globalización, por ejemplo, puede provocar una amplificación o una atenuación del efecto de las fuerzas que impulsan el cambio sobre los usos del suelo (Lambin y Geist, 2006). La identificación de estos factores promotores del cambio es uno de los prerrequisitos para desarrollar un modelo de cambios de usos del suelo realista (Veldkamp y Lambin, 2001). Los análisis de cambios de usos sirven para monitorear cambios en los cultivos, valorar el impacto de deforestaciones, estudiar cambios en la fenología de la vegetación, detectar el estrés hídrico de cultivos, etc. La transformación de estos cambios por la acción humana puede afectar la integridad de los recursos naturales y la producción de bienes y servicios y, por ende, su análisis debería ser una parte fundamental para el planeamiento de un desarrollo sostenible (Gallardo y Martínez-Vega, 2016).

La generación de escenarios futuros contribuye, además, a un mejor entendimiento de estos procesos. Pueden ser utilizados como un sistema de alerta temprana que manifiesta y subraya los efectos de los cambios futuros de usos del suelo y los puntos calientes que son prioritarios para un análisis en profundidad o con interés en la intervención política (Verburg et al., 2006a). No deben ser tomados como una predicción ni un pronóstico, sino como descripciones e imágenes coherentes, consistentes y plausibles de un posible estado futuro o, como Alcamo et al. (2006) señalan, de "futuros no inverosímiles", que ayuden a manejar las incertidumbres, examinando varias alternativas de futuro y comparando las consecuencias potenciales que éstos pueden tener (Verburg et al., 2006c).

El desarrollo de las diferentes técnicas que permiten elaborar modelos de cambios de usos del suelo y/o de simulaciones a futuro ha estado directamente relacionado con el avance de la teledetección, de los sistemas de información geográfica y de nuevos softwares que han permitido la captura y el tratamiento de grandes conjuntos de datos. 
Investigadores, científicos y planificadores han prestado mucha atención a estos aspectos en las últimas décadas. Se han desarrollado numerosos trabajos usando diferentes metodologías y algoritmos, tratando de observar los patrones emergentes de los diferentes procesos de cambios de los usos del suelo; sin embargo, el componente geográfico, tan diferente y variado, hace difícil desarrollar una metodología general para poder aplicarla en diferentes regiones de la Tierra. Existe un amplio mosaico de distintas técnicas y modelos cada vez más complejos. Son numerosos los estudios y proyectos cuyo objetivo es la creación de escenarios en relación con la valoración de la sostenibilidad. La sostenibilidad tiene implicaciones muy diversas, por lo que dichos estudios son también muy variados dependiendo del objetivo que se pretende alcanzar.

Este trabajo muestra una recopilación de trabajos internacionales y nacionales (en España) sobre modelos de cambios de usos del suelo y de simulaciones a futuro que se han desarrollado en los últimos años, definiendo los diferentes conceptos de los que se hace uso y los avances que se han realizado. La primera parte muestra la tipología de modelos que existen diferenciándolos según seis características diferentes. Posteriormente, se detallan las metodologías desarrolladas para el estudio de los cambios de usos del suelo y los factores explicativos más utilizados (epígrafe 3 y 4 , respectivamente). El epígrafe 5 se centra en los estudios sobre escenarios de usos del suelo a futuro aplicados en diferentes ámbitos geográficos. Finalmente, se describen una serie de conclusiones y se debate acerca de los problemas y retos a los que se enfrenta esta disciplina.

\section{Grandes tipologías de modelos de cambios de usos del suelo}

En el estudio de los cambios de usos del suelo se pueden diferenciar varios tipos de modelos. Una primera distinción que puede hacerse es entre modelos no espaciales y modelos espaciales. Algunos modelos se centran en predecir tasas o cantidades de cambio, mientras que otros ponen más énfasis en los patrones espaciales. El cambio puede estar indicado por píxeles individuales o por otras entidades espaciales, tales como entidades poligonales o unidades administrativas. Cada vez son más los modelos que tratan de hacer frente a estos dos temas de una manera integrada.

Una segunda distinción puede hacerse entre modelos dinámicos y modelos estáticos. Los primeros otorgan una atención especial a las dinámicas temporales del sistema de usos del suelo, representados por la competición entre los usos del suelo, la dependencia de los cambios pasados y las trayectorias de cambios de usos establecidas. Los modelos estáticos pueden ser utilizados para testear nuestro conocimiento acerca de los factores explicativos del cambio, mientras que los modelos dinámicos son utilizados para realizar proyecciones futuras de cambios de usos (Verburg et al. 2006a). 
Una tercera distinción de modelos se da entre modelos deductivos y modelos inductivos. La mayoría de los modelos de usos del suelo tienen un enfoque inductivo, en el cual las especificaciones del modelo están basadas en correlaciones estadísticas entre los cambios de usos y un conjunto de variables que proporcionan una idea de este cambio. Existen diferentes tipos de modelos inductivos, desde modelos donde la toma de decisiones la llevan a cabo diferentes actores y donde las interacciones están basadas en observaciones, hasta modelos donde la relación entre los patrones de usos y la variabilidad espacial de las condiciones socio-económicas y biofísicas se captura mediante técnicas estadísticas, normalmente regresiones o redes neuronales. Los modelos deductivos, en cambio, están basados en la teoría que predice patrones desde los procesos; utilizan la teoría para caracterizar las relaciones de los patrones de usos del suelo; estructuran al modelo mediante las relaciones entre el hombre y el medio y centran la atención en los datos requeridos para explorar dichas relaciones (Verburg et al. 2006a).

Los modelos pueden ser también cualitativos y/o cuantitativos. Los primeros incorporan situaciones pasadas, presentes o posibles futuros en forma de palabras en lugar de números; pueden tomar la forma de imágenes, diagramas, frases o esquemas, pero en la realización de escenarios lo más frecuente es que se compongan de textos narrativos, comúnmente llamados "storylines"; estos storylines pueden representar opiniones y visiones de las partes interesadas y de los expertos al mismo tiempo, desarrollándose a través de un enfoque participativo, donde ambos trabajan juntos en el desarrollo de estos escenarios. Los modelos cuantitativos, sin embargo, suelen ser llevados a cabo mediante procesos informáticos y su resultado nos proporciona información numérica en forma de tablas, gráficos y mapas (Alcamo et al., 2006). En este sentido, los modelos cuantitativos pueden tener como unidad de representación los píxeles (representados espacialmente en un ráster) u otras entidades como parcelas catastrales o unidades administrativas (representados espacialmente en un formato vectorial). Los modelos cualitativos están basados en modelos de agente, que a su vez pueden quedar representados sobre unidades de tipo píxel o vectoriales, combinando, por tanto, las dos tipologías de modelos.

Por último, a nivel espacial, podemos distinguir entre modelos locales y regionales y modelos globales. Los dos primeros son los más numerosos, mientras que se han desarrollado muy pocos modelos globales de cambios de usos del suelo. Esto es debido a que el estudio de la evolución de los cambios de usos de la tierra a nivel global es limitado, ya que la mayoría de los procesos que influyen en el cambio global son el resultado de las decisiones y cambios que se dan a escala local o regional y que, por tanto, están representados de manera pobre en las evaluaciones a escala global (Verburg et al., 2006b). La cuestión básica que desean abordar también es diferente: los escenarios globales tienden a responder cuánto uso del suelo cambiará, normalmente a largo plazo (dentro de 50 años o más), mientras que los escenarios regionales y locales tienden a responder también dónde tendrá lugar el cambio, habitualmente en un corto plazo (en los próximos 20 o 30 años). A pesar de que los escenarios a escala regional y local son más variados y más numerosos que los globales, a día de hoy existe 
un incipiente aumento de estos últimos (Malek et al., 2018). Escasos o muy escasos son los modelos multi-escalares, integrando, por ejemplo, la escala europea con la nacional, o la escala nacional con la local. Esto tiene un gran interés, puesto que los modelos locales o a nivel de paisaje son adecuados para hacer frente a las condiciones específicas de un área de estudio determinada, pero suelen ser incapaces de dar cuenta de los procesos a escalas más groseras. Un ejemplo de este tipo de estudios puede verse en el artículo de Verburg et al. (2006c) donde realizan conjuntamente cuatro escenarios diferentes a escala de la Unión Europea y a escala estatal.

Los cambios de usos del suelo están influenciados por una gama de factores locacionales específicos. La adquisición y comprensión de estas variables explicativas que determinan procesos humanos y naturales es mucho más amplia a nivel de detalle y de ellas deriva el buen funcionamiento y resultado de los modelos. En este sentido, Titeux et al. (2017) señalan que los modelos de cambios y de simulaciones locales y regionales deberían ser incorporados en modelos de cambio climáticos o de biodiversidad realizados también a escalas globales.

\section{Metodologías para el análisis de cambios de usos del suelo}

A la hora de cuantificar los cambios de usos del suelo, la mayor parte de los trabajos se han fundamentado y se fundamentan hoy en día en una matriz de transición o también llamada matriz de tabulación cruzada, la cual nos permite cruzar dos datos temporales representados por píxeles y obtener valores de cambio total, cambio neto, intercambio, ganancias brutas y pérdidas brutas (Bell, 1974; Pontius et al., 2014). Sin embargo, en la última década son varios los autores que consideran que la medición de los cambios de usos del suelo no puede realizarse únicamente mediante un análisis de píxel a píxel. Así, Hagen-Zanker (2006) propone un método de una ventana movible, ponderando la distancia, basándose en la idea de que la estructura en la localización de los usos está definida por su vecindad y las celdas cercanas al centro de dicha vecindad tienen una influencia mayor que las que se localizan en los bordes. Otro numeroso grupo de expertos (Rae et al., 2007; García et al., 2012; Aguilera-Benavente et al., 2014) sugiere la incorporación diferentes métricas del paisaje para cuantificar también cambios en la estructura del paisaje.

El análisis de los cambios de usos del suelo no puede ser una mera descripción de los valores cuantitativos de cambio. Es necesario conocer los factores explicativos que impulsan estos cambios, los cuales son fundamentales para poder generar modelos de usos futuros. Con este fin se han ido desarrollando a lo largo del tiempo diferentes metodologías, muchas de las cuales hoy en día se combinan entre sí.

McDonald y Urban (2006) comentan que los primeros modelos de cambios de usos y cubiertas del suelo eran no-espaciales, donde la probabilidad de cambio para todas 
las zonas estaba estimada como la proporción de celdas que habían cambiado en el último periodo de tiempo; esto describe un proceso de cadenas de Markov (MC), donde la probabilidad de un cambio en una cubierta de suelo (por ejemplo, de bosque a no bosque) es constante e igual para todas las áreas de bosque. Así, las cadenas de Markov permiten analizar los cambios cuando los datos no son espacialmente explícitos o son de una resolución espacial grosera, y permiten, a su vez, la exploración inductiva del cambio de usos del suelo proporcionando pistas sobre la dinámica de éstos. Para estimar el futuro, asumen que el pasado inmediato es el mejor predictor del futuro próximo, bajo la condición de estacionalidad (Geoghegan et al., 1998).

Las probabilidades de transición de Markov deben hacerse espacialmente explícitas, ya que la localización de los cambios de usos del suelo es un dato esencial a tener en cuenta. Es por ello que, posteriormente, se incorporan un conjunto de variables explicativas que sí pueden ser modeladas espacialmente, como el definido por McDonald y Urban (2006) mediante la ecuación:

$$
P(d) i=f(x i)+\varepsilon
$$

donde, la probabilidad de cambio en el píxel $i$ es una función del vector de variables explicativas medidas en la localización $i$. Esta función puede ser tan complicada como se desee, dependiendo de las variables explicativas contenidas en $x$. $x$ puede contener información del estado de los píxeles adyacentes, como es la proporción de celdas vecinas que han sido ya deforestadas, proporcionando al modelo atributos de un autómata celular (CA), en el que los procesos de simulación se basan en la interacción de los píxeles vecinos, definiendo el dominio geográfico de influencia (Jokar et al., 2013); de esta manera, el cambio no sólo depende de su estado previo, sino también del estado previo de sus vecinos inmediatos. Los CA fueron desarrollados por von Neumann y Ulam y el concepto, en relación con los modelos de simulación de cambios de usos del suelo, es introducido por Tobler (1970). En los últimos años estos modelos basados en CA han proliferado y se han convertido en una de las técnicas más utilizadas para modelar las dinámicas complejas presentes en los sistemas urbanos (Santé et al., 2010) y poder predecir el crecimiento urbano.

Muchas de las reglas que gobiernan los cambios de usos y cubiertas del suelo varían de un sitio a otro. Hay fuerzas exógenas como decisiones políticas, zonificaciones o el deseo de determinados agentes en localizar cierto uso en un sitio determinado, que pueden tener profundos efectos en los cambios de usos del suelo. Estas fuerzas alteran las trayectorias de los cambios y sugieren que la función difiere dependiendo del lugar: las reglas del cambio son, pues, espacialmente heterogéneas. Hay que tener en cuenta, además, que la relación entre los cambios de usos del suelo y las variables no siempre se establece de manera lineal (McDonald y Urban, 2006). En un modelo de regresión logística (RL) la probabilidad de cambio se define como:

$$
\ln \left(\frac{p}{1-p}\right)=\beta_{0}+\beta x+\varepsilon
$$


Donde $p$ es la probabilidad de cambio, $\beta$ son los parámetros estimados y $x$ es el vector de las variables explicativas. Una regresión logística es una forma específica de un modelo lineal generalizado (GLM). Es un ejemplo de modelo espacialmente homogéneo. Sin embargo, si las variables explicativas y las múltiples interacciones son espacialmente estructuradas es posible obtener un porcentaje de heterogeneidad espacial en las reglas de cambio de usos.

Un ejemplo de modelos espacialmente heterogéneos pueden ser los árboles de clasificación (CART) o las redes neuronales (Geoghegan et al., 1998). Los CART presentan un enfoque no paramétrico que parte, de forma recursiva, de un conjunto de datos en subconjuntos que son cada vez más homogéneos con respecto a la variable respuesta, basándose en una división binaria óptima de un conjunto de variables explicativas (Moore et al., 1991). Las redes neuronales (RN) son capaces de modelar el cambio y cuantificar el comportamiento complejo y los patrones de cambio teniendo en cuenta relaciones no lineales entre las variables explicativas y los cambios de usos del suelo y el carácter correlacionado de las variables, tanto de naturaleza cronológica como espacial (Paegelow et al., 2003; Vafeidis et al., 2007); consiste en capas y neuronas que simulan la estructura de un celebro humano: consta de una capa de entrada, una capa de salida y unas capas ocultas entre ambas; las neuronas o nodos son las unidades básicas del proceso y están dispuestas en dichas capas (Li y Yeh, 2002).

En los modelos basados en agentes o actores (ABM) o en sistemas multiagentes (MAS), la decisión de éstos es análoga a la función descrita previamente; si existen diferentes tipos de agentes con diferentes preferencias habrá entonces múltiples funciones para cada una de las tipologías de agente (McDonald y Urban, 2006). Estos modelos representan las decisiones humanas y sus interacciones. Su importancia radica en la pauta de que el territorio es un medio donde los actores interactúan, por lo que es importante conocer las reglas que definen las relaciones entre los agentes y el medio, y las reglas que determinan el orden de las acciones en el modelo; el actor (un individuo, un hogar o una institución) lleva a cabo acciones específicas de acuerdo a sus propias reglas de decisión, lo que deriva en cambios en las cubiertas de la tierra. Estas decisiones son las que deben ser parametrizadas por las reglas de decisión (Parker et al., 2002; Valbuena et al., 2008). Una de las desventajas de la utilización de los agentes como unidad básica de simulación es la dificultad de representar adecuadamente el comportamiento y la complejidad de las interacciones humanas y medioambientales a diferentes escalas espaciales (Rindfuss et al., 2004; Rounsevell et al., 2012).

Otro método utilizado es la Evaluación Multicriterio (EMC) que considera la capacidad del territorio para acoger un determinado uso del suelo y no sólo el ritmo temporal de éste. Mediante este método pueden ser definidas las reglas de transición y puede generarse un mapa de idoneidad, en función de unos objetivos y criterios establecidos (Paegelow et al., 2003).

Los modelos de cambios de usos no tienen por qué utilizar una única técnica de modelización como las aquí descritas, sino que muchas veces combinan varias (tabla 1). 


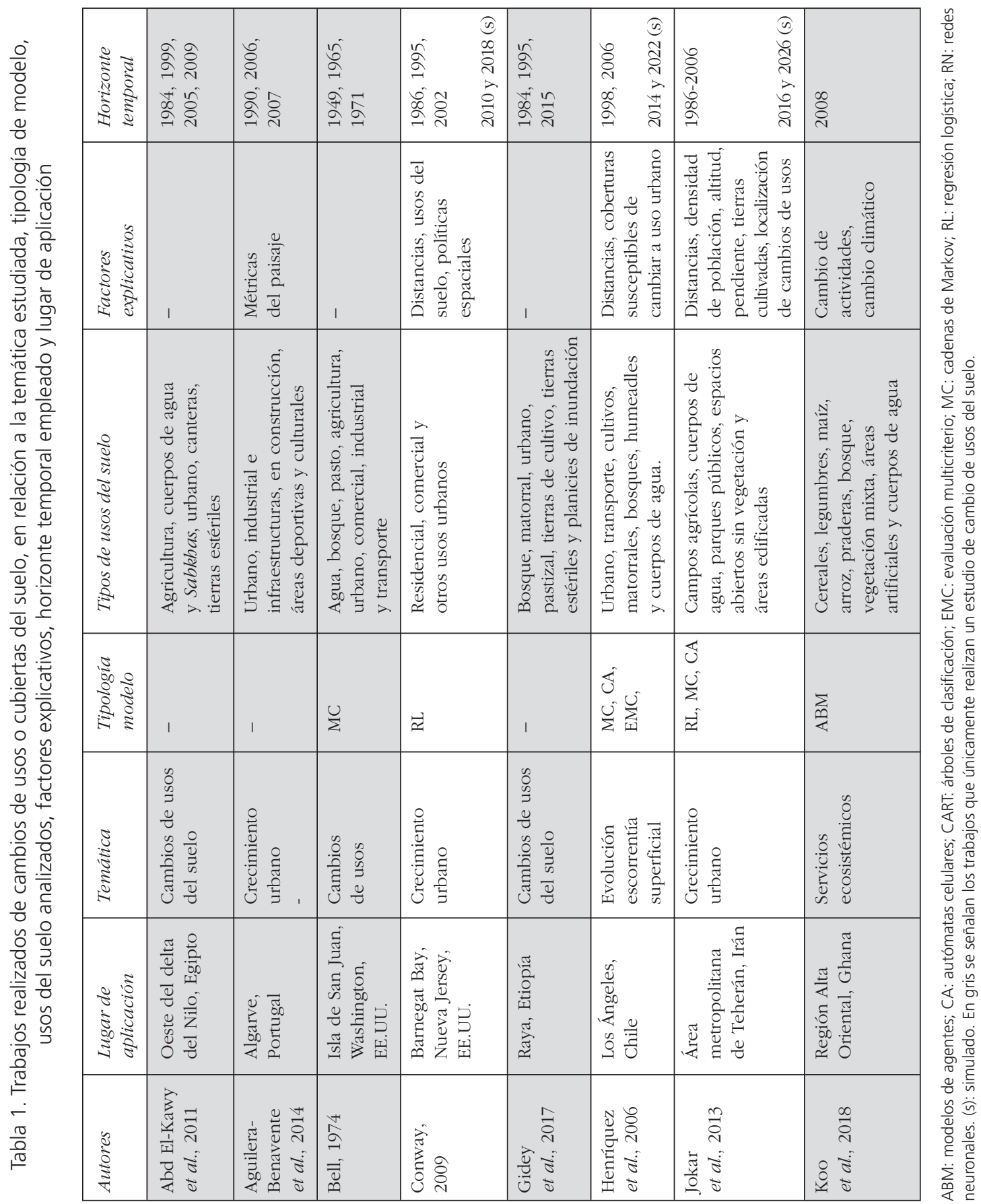




\begin{tabular}{|c|c|c|c|c|c|}
\hline 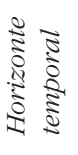 & 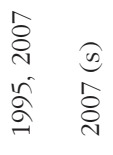 & 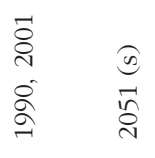 & $\begin{array}{l}\approx \\
\stackrel{2}{\sigma} \\
\frac{1}{\infty} \\
\infty \\
\sigma \\
\sigma\end{array}$ & 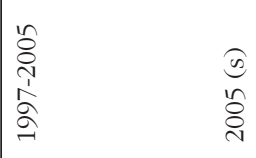 & 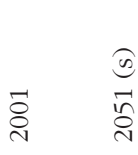 \\
\hline 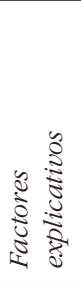 & 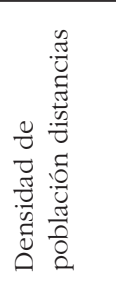 & 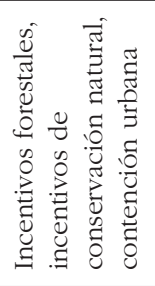 & 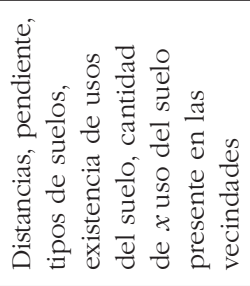 & 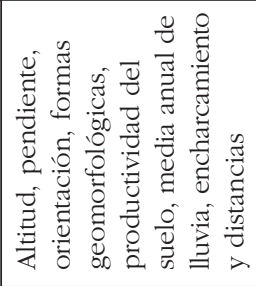 & 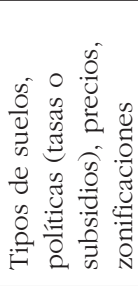 \\
\hline $\begin{array}{l}0 \\
3 \\
0 \\
0 \\
8 \\
0 \\
0 \\
3 \\
0 \\
0 \\
0 \\
0 \\
0\end{array}$ & 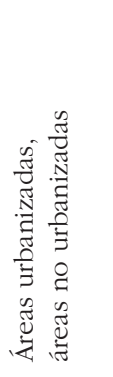 & 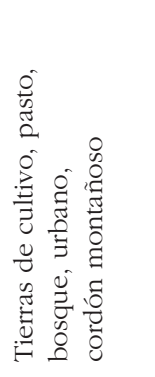 & 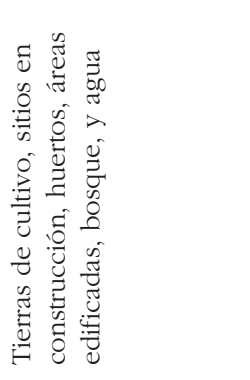 & 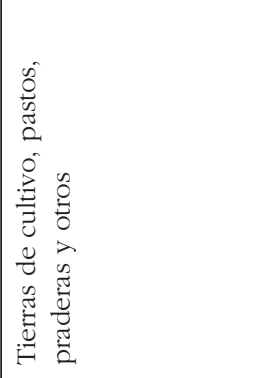 & 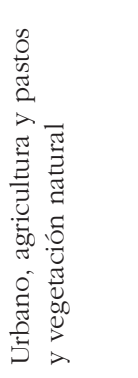 \\
\hline 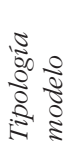 & 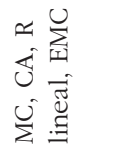 & 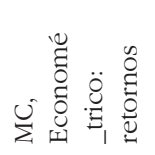 & $\begin{array}{l}Z \\
Z \\
\lambda \\
\sum_{9}\end{array}$ & $\vec{x}$ & 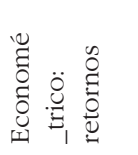 \\
\hline 离 & 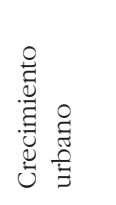 & 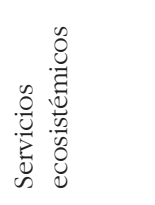 & 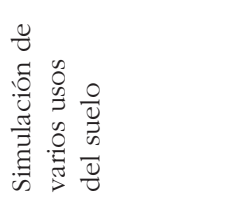 & 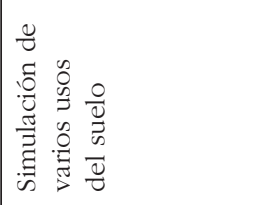 & 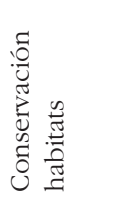 \\
\hline 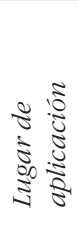 & 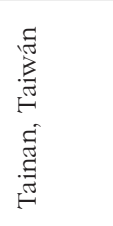 & 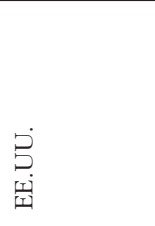 & 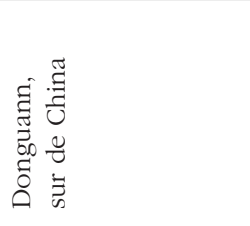 & 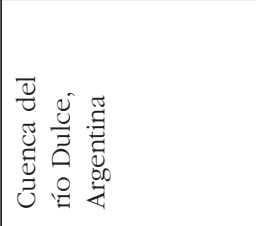 & 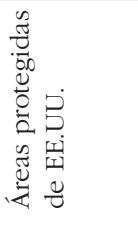 \\
\hline 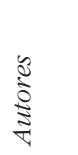 & $\begin{array}{l}0 \\
\stackrel{1}{2} \\
\vec{\nabla} \\
\overrightarrow{1}\end{array}$ & 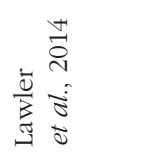 & 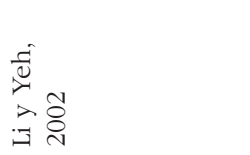 & 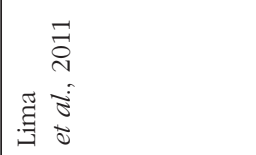 & 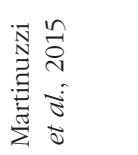 \\
\hline
\end{tabular}




\begin{tabular}{|c|c|c|c|c|c|}
\hline 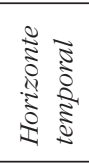 & 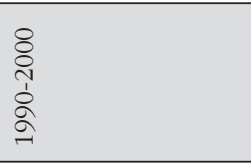 & 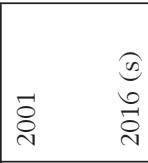 & 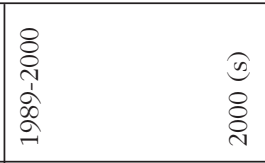 & 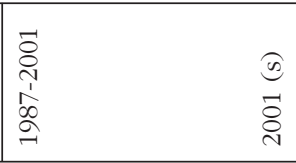 & 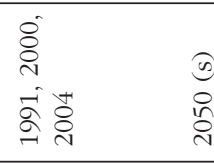 \\
\hline 这 & 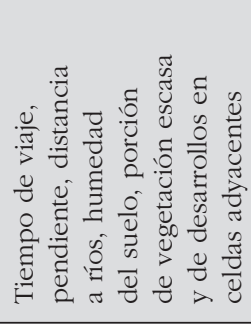 & 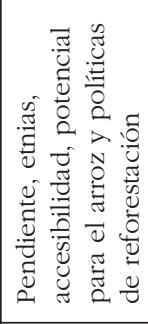 & 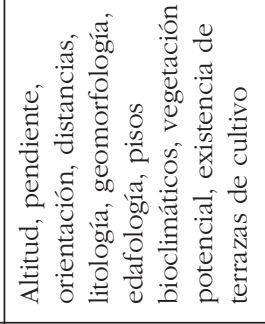 & & 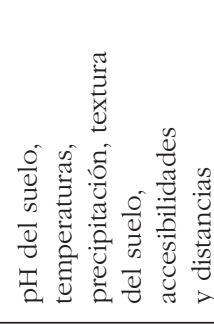 \\
\hline 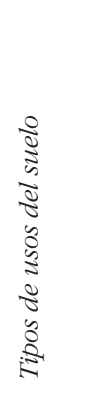 & 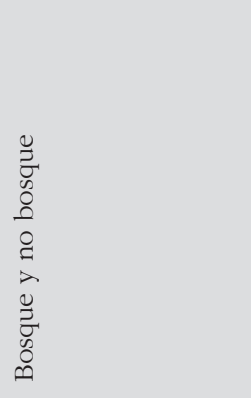 & 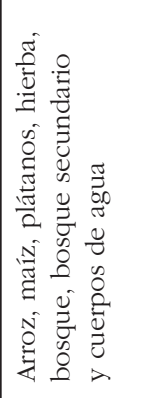 & 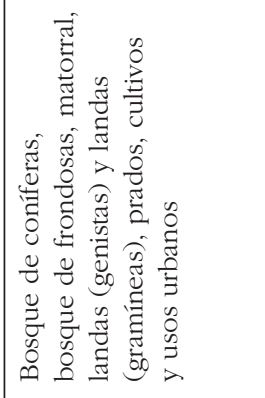 & 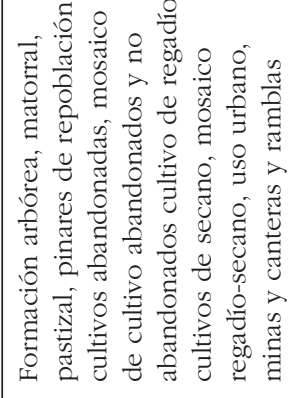 & 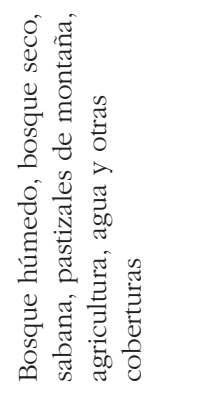 \\
\hline 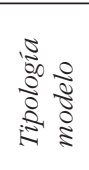 & 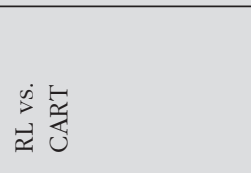 & 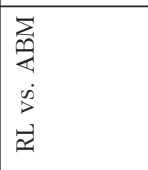 & \multicolumn{2}{|l|}{ 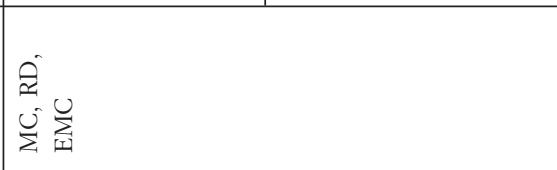 } & $z$ \\
\hline 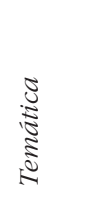 & 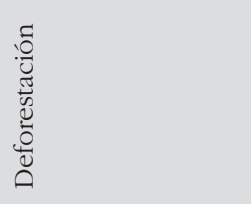 & 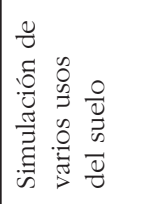 & \multicolumn{2}{|l|}{ 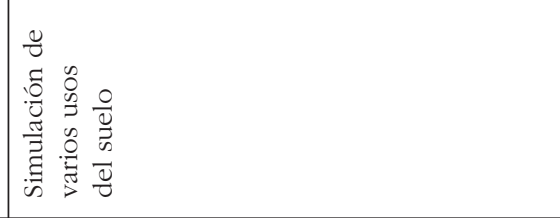 } & 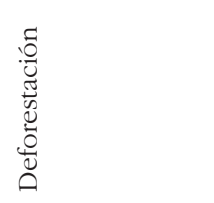 \\
\hline 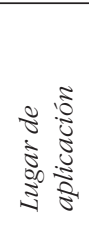 & 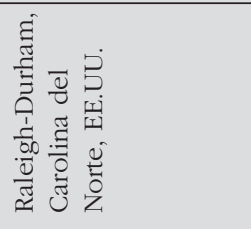 & 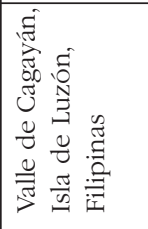 & 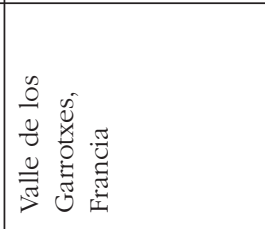 & 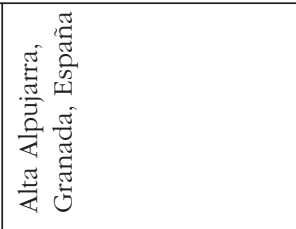 & 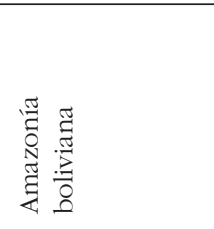 \\
\hline 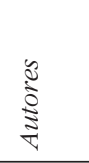 & 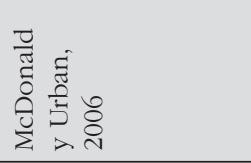 & 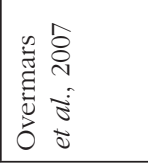 & \multicolumn{2}{|l|}{ 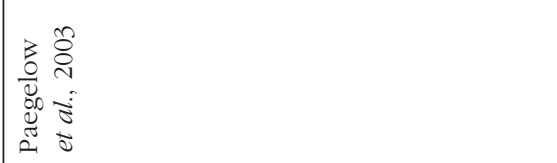 } & 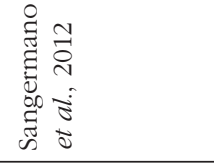 \\
\hline
\end{tabular}




\begin{tabular}{|c|c|c|c|}
\hline 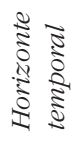 & 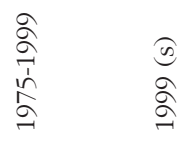 & 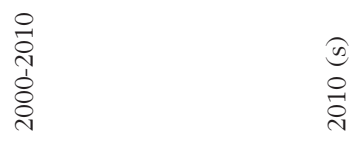 & ڤึ) \\
\hline 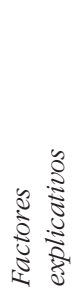 & 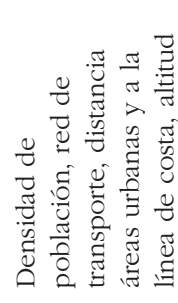 & 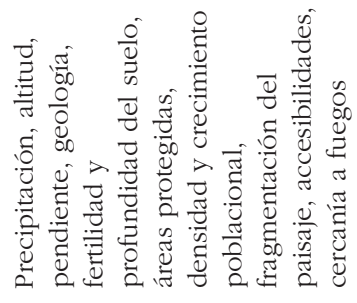 & 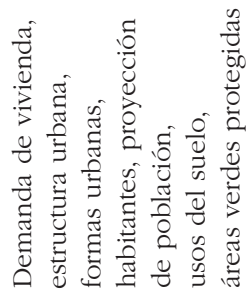 \\
\hline 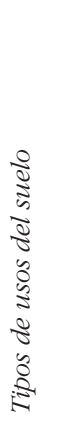 & 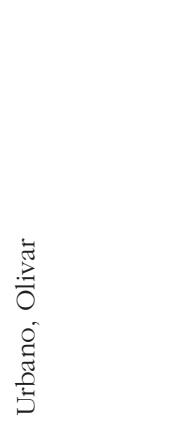 & 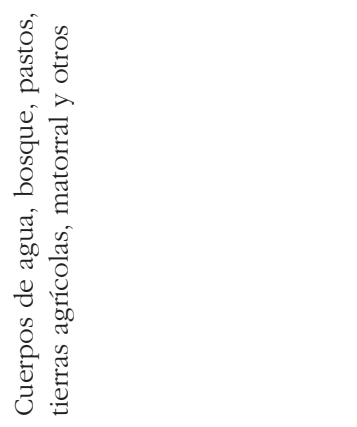 & 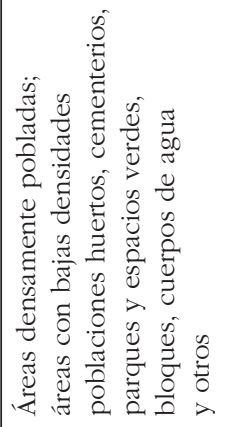 \\
\hline 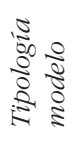 & $\not \check{x}$ & $\vec{z}$ & 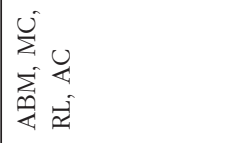 \\
\hline 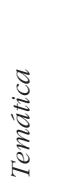 & 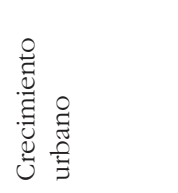 & 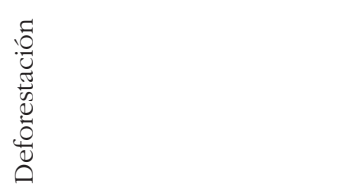 & 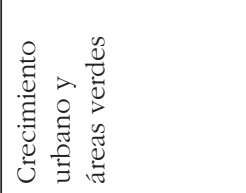 \\
\hline 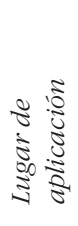 & 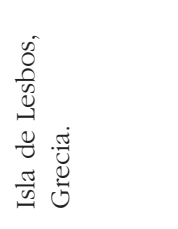 & 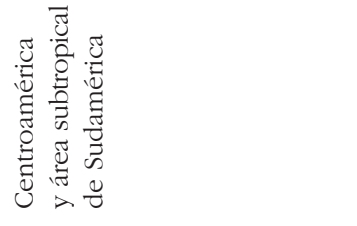 & 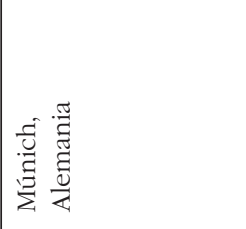 \\
\hline$\frac{3}{3}$ & 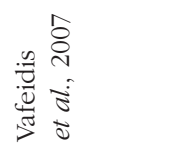 & 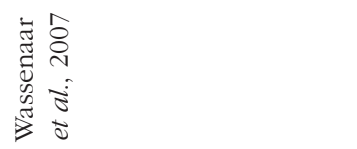 & $\begin{array}{l}\overrightarrow{0} \\
\overrightarrow{0} \\
\overrightarrow{0} \infty \\
\vec{x} \\
\vec{a}\end{array}$ \\
\hline
\end{tabular}

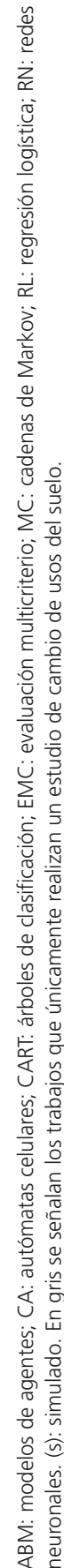




\section{Selección de factores o variables explicativas}

La selección de los factores explicativos de los cambios de usos del suelo que se desean analizar es fundamental, ya que de ella dependerá el resultado de nuestro modelo. Un modelo debe tratar de combinar factores biofísicos y humanos que traten de explicar los patrones espaciales de los diferentes usos del suelo con los que se trabaja. El objetivo del estudio, la escala espacial y temporal y las particularidades de la zona que se desea modelar determinarán la elección de dichos factores, pues éstos están directamente relacionados con el tipo de patrón que será observado; es decir, los patrones que aparecen a un nivel de resolución determinado no tienen por qué observarse a una resolución mayor o menor.

Los factores biofísicos están determinados por factores naturales que condicionan la localización de los cambios de usos; éstos pueden ser: climatológicos, litológicos, topográficos, hidrológicos, vegetacionales, etc. Los cambios de usos están condicionados, a su vez, por factores socio-económicos como pueden ser el empleo, las migraciones, los mercados, los costes de transporte, etc.; los factores culturales también tienen un papel importante: las motivaciones, actitudes o tradiciones afectan a las decisiones de localización.

A pesar de que se deben considerar factores naturales y socio-económicos en su conjunto, no todos los estudios llevan a cabo un análisis de ambos. Prácticamente todos los trabajos utilizan como variables las derivadas de un modelo digital del terreno, es decir, altitud y pendiente y, en muchos casos, la orientación también. Igualmente, son considerados factores como la temperatura o las precipitaciones o datos geológicos, litológicos o geomorfológicos. Son numerosos también los estudios que incluyen factores de accesibilidad, sea en modo de distancias o de costes de transporte. Factores demográficos, como pueden ser la densidad de población, también son muy utilizados, así como datos de empleo en determinados sectores económicos (tabla 1).

Huelga decir que la política gubernamental juega un papel decisivo en los cambios de usos; para Rounsevell et al. (2012) los usos del suelo futuros están fuertemente determinados por cambios en las políticas, como pueden ser reformas en las políticas agrarias, la liberación de los mercados o la conservación de la naturaleza, pero también con políticas de mitigación o adaptación al cambio climático. Verburg et al. (2004a) comentan que cualquier estudio que trate sobre cambios en los patrones de usos del suelo debería estar precedido por un inventario de las políticas espaciales que son relevantes en el área de estudio.

Así, se deben tener en cuenta los patrones históricos de los usos del suelo, las restricciones climáticas, económicas y ecológicas, las causas del cambio, cómo las diferentes causas actúan juntas creando sinergias y cómo las diferentes actividades retroalimentan y afectan a dichas causas (Reid et al., 2006). 
Cualquier descripción del sistema de usos del suelo sin un componente humano dinámico nunca alcanzará un valor realista (Veldkamp y Fresco, 1997), en especial, cuando se estudia un periodo de tiempo corto a escala local, ya que, en este ámbito, son los factores humanos los que normalmente dominan las fuerzas del cambio.

La preparación de estos datos de entrada es una tarea muy importante. La figura 1 muestra un ejemplo del funcionamiento de un modelo espacialmente explícito donde, mediante la utilización de factores explicativos de la cantidad de cambio y de factores explicativos de la localización del cambio, puede desentrañarse la magnitud del cambio y generar un mapa de idoneidad del cambio.

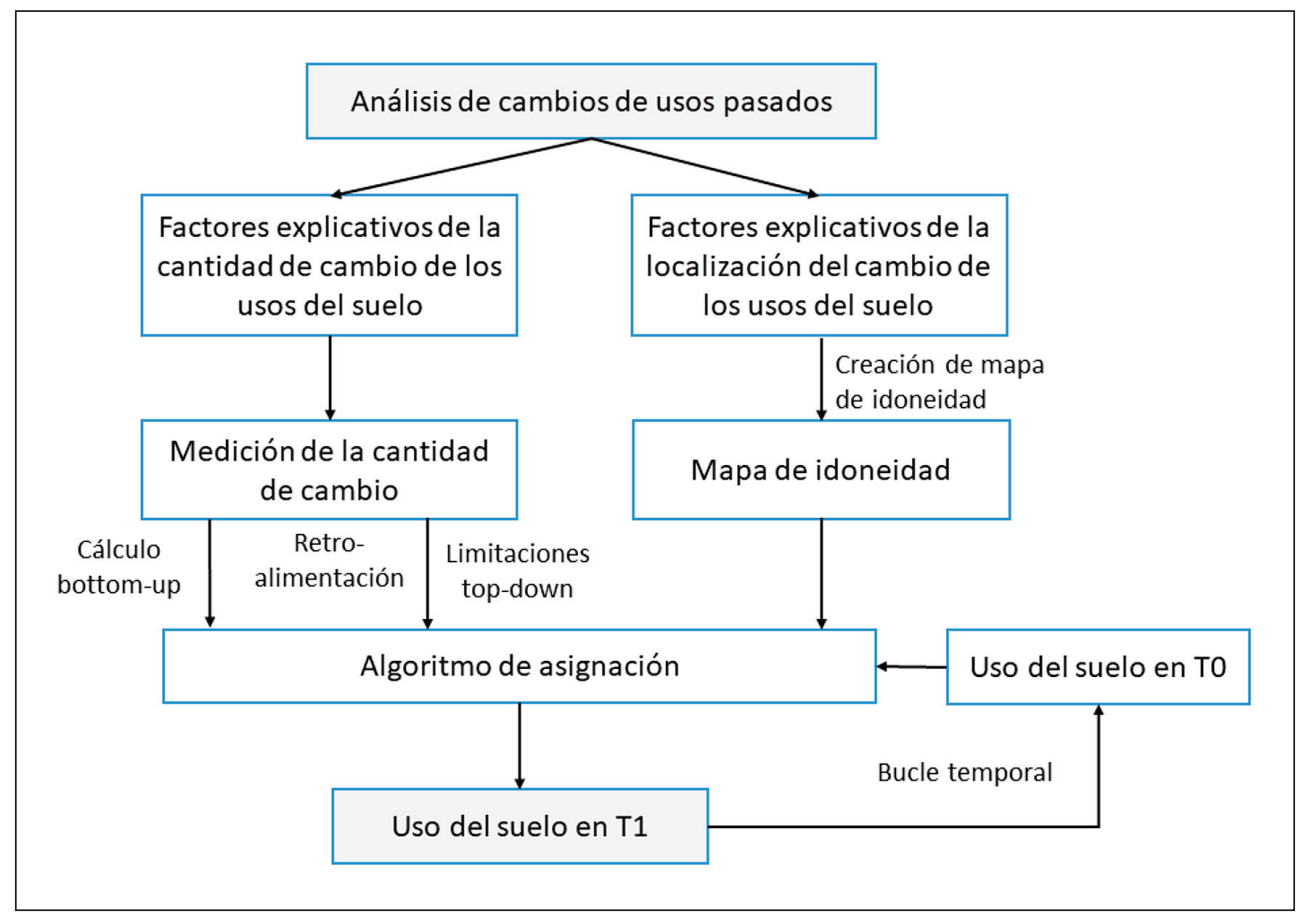

Figura 1. Funcionamiento de los modelos espacialmente explícitos.

Elaboración propia a partir de Lambin y Geist (2006).

\section{Simulación de escenarios a futuro}

Dependiendo del fin que se desea alcanzar mediante la creación de escenarios futuros, dependerá la escala espacial y temporal a utilizar, la tipología de usos del suelo a escoger, la metodología y las variables explicativas, etc. La disponibilidad de datos 
de partida es, en ocasiones, la que determina que un estudio pueda llevarse a cabo o no. Se pueden definir diferentes escenarios de futuro, según las trayectorias de futuro que podrían darse o las estrategias de planificación que se desean implementar.

Los estudios de escenarios comprenden desde escalas globales a escalas locales, si bien, los primeros son los más escasos en cuanto a número. A nivel de continente o región, entendida ésta como una escala que comprende varios países, destacan en número los desarrollados en Europa o a escala de la Unión Europea, muchos de los cuales están basados en el IPCC (Intergovernmental Panel of Climate Change), antiguamente con las storylines del Special Report on Emision Scenarios (SRES) y a partir de 2014 con las trayectorias previstas en el Representative Concentration Pathways (RCP).

La mayoría de los escenarios muestran los cambios que se han producido y se producirán en los usos agrícolas, ya que éstos han sido los usos más dinámicos, seguidos de los escenarios forestales que son, en gran medida, un reflejo de los escenarios agrícolas. Si bien, en los últimos años se ha mostrado un creciente interés por los cambios de usos del suelo urbanos (Deng y Srinivasan, 2016; Romano et al., 2017).

Numerosas investigaciones de simulaciones de cambios de usos del suelo se centran en temas de deforestación, en relación con la expansión de la agricultura o de los pastizales, o el crecimiento y la dispersión urbana. La conservación de la biodiversidad es otro de los objetivos recurrentes, en especial los efectos que los cambios de usos del suelo pueden tener en el hábitat de las especies animales y/o vegetales, sus efectos en el ciclo hidrológico o en la erosión o en los servicios ecosistémicos. En relación con esto, un gran número de estudios de escenarios también se centran en métricas del paisaje, con el objetivo de valorar cómo pueden influir los cambios de usos del suelo en índices como la fragmentación, la conectividad o la diversidad.

La elaboración de escenarios a nivel local se ha sucedido por toda la superficie de la Tierra. La tabla 1 muestra un ejemplo de 20 investigaciones internacionales que utilizan diferentes técnicas y modelos para analizar los cambios pasados y/o futuros en áreas urbanas de China, Chile o Irán, en espacios naturales protegidos en EE.UU o en cuencas hidrográficas de Egipto o Argentina.

A nivel estatal, en España, son variados los trabajos de cambios de usos del suelo, y en menor medida, aunque con un interés creciente, de escenarios a futuro. La tabla 2 recoge un ejemplo de estos estudios localizados en provincias variadas como Girona, Granada, Lugo, Madrid o Tenerife, muchos de los cuales se centran en análisis históricos y/o prospectivos de dinámicas urbanas. 
Revisión y análisis de estudios de modelos de cambios de usos del suelo... Geographicalia, 70, 2018: 1-26

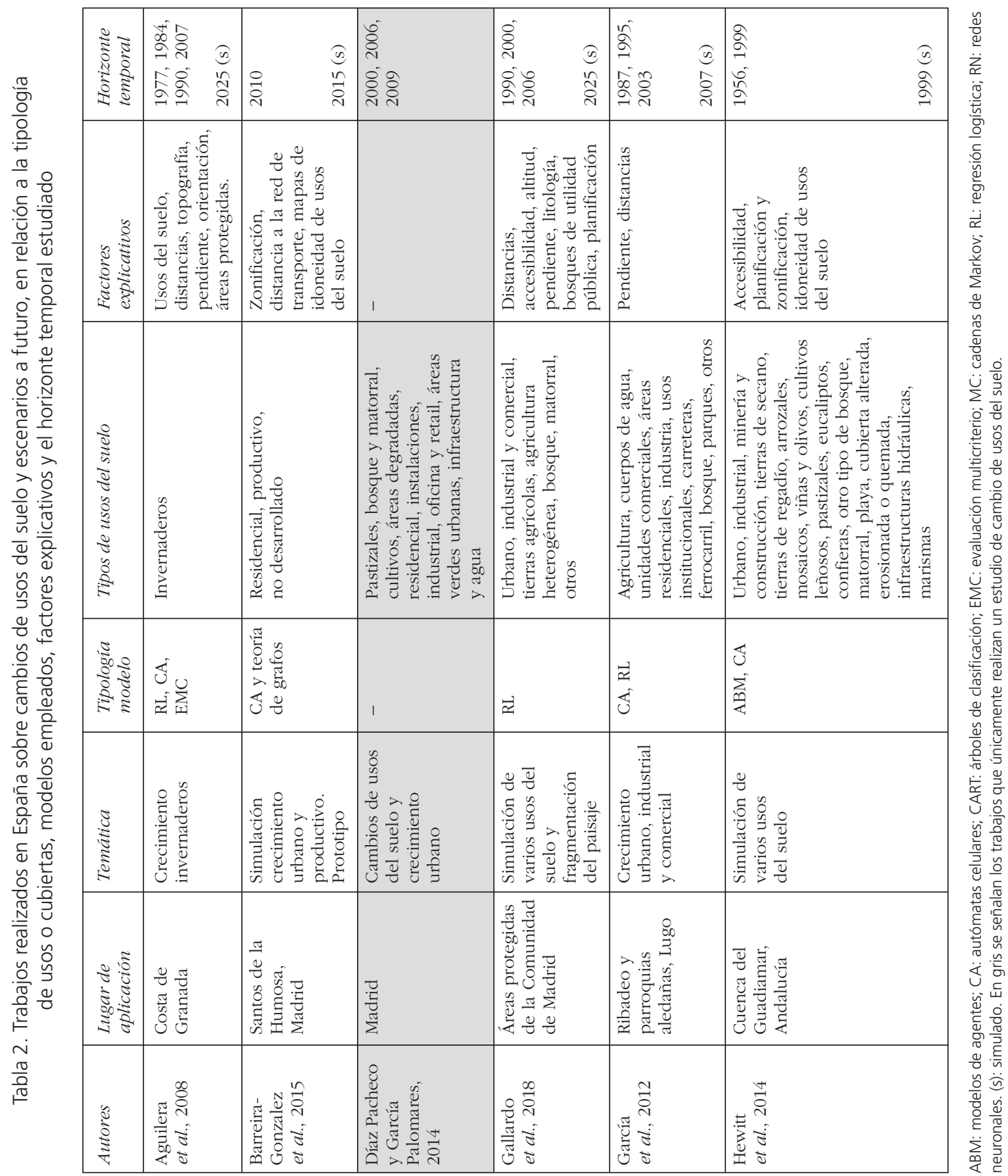




\begin{tabular}{|c|c|c|c|c|}
\hline 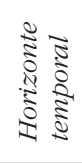 & $\begin{array}{l}\text { (3) } \\
\text { oे } \\
\text { ते }\end{array}$ & \begin{tabular}{l}
8 \\
$\stackrel{8}{1}$ \\
1 \\
10 \\
2 \\
\hdashline \\
-1
\end{tabular} & 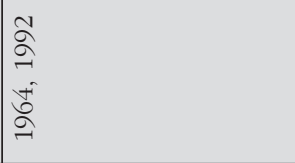 & 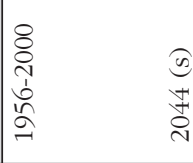 \\
\hline 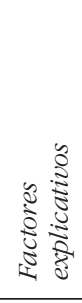 & 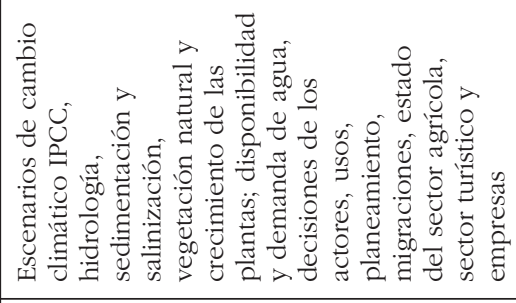 & 1 & 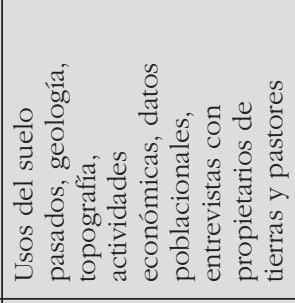 & 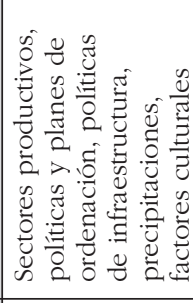 \\
\hline 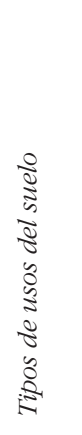 & 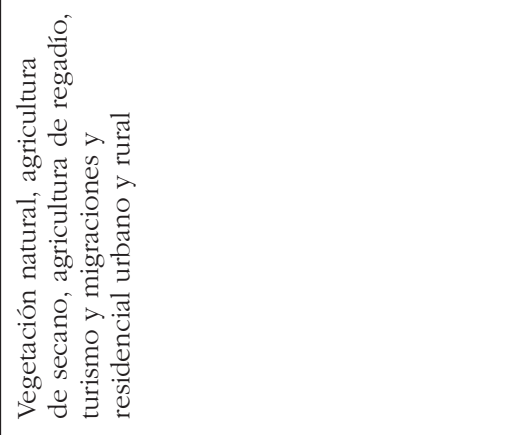 & 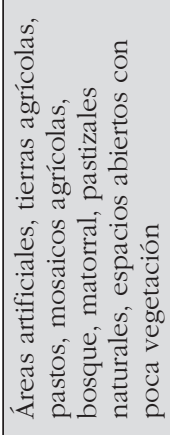 & 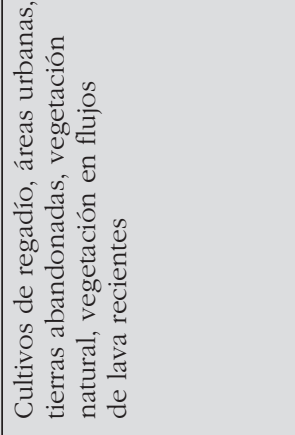 & 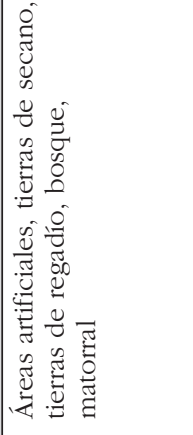 \\
\hline 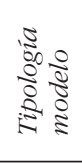 & 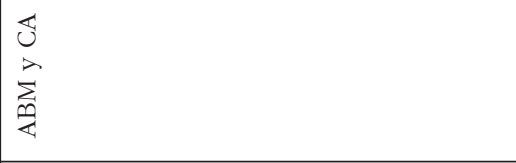 & 1 & 1 & $\frac{U}{z}$ \\
\hline 芯 & 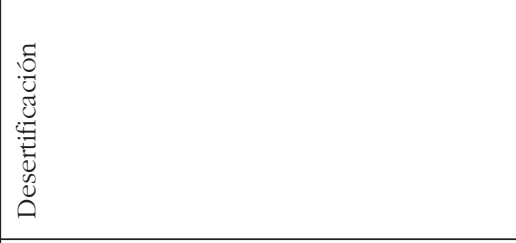 & 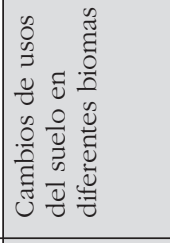 & 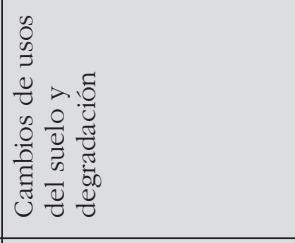 & 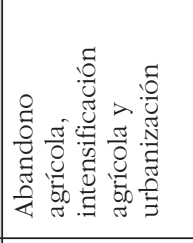 \\
\hline 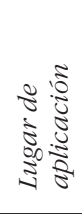 & 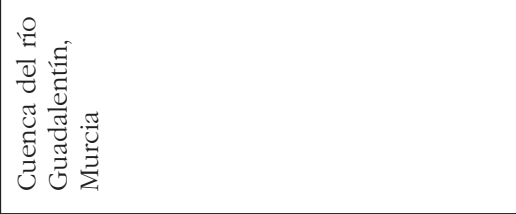 & 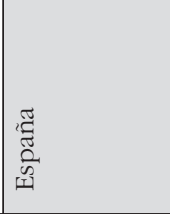 & 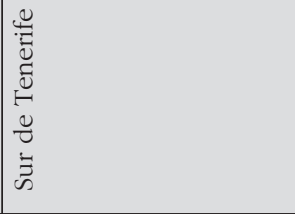 & 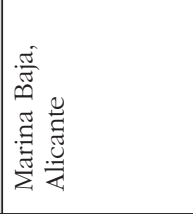 \\
\hline 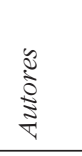 & 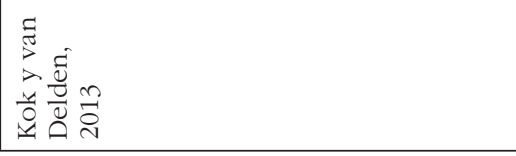 & 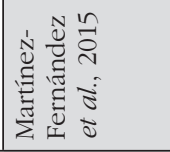 & 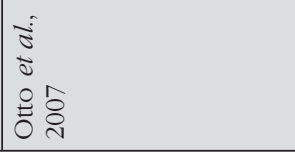 & 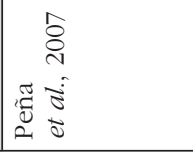 \\
\hline
\end{tabular}




\begin{tabular}{|c|c|c|c|c|}
\hline 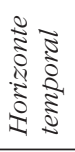 & 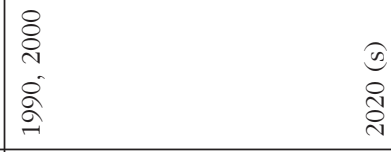 & 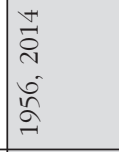 & 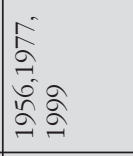 & 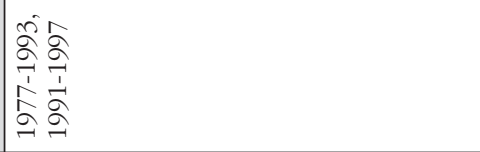 \\
\hline 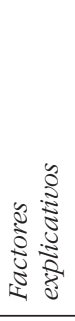 & 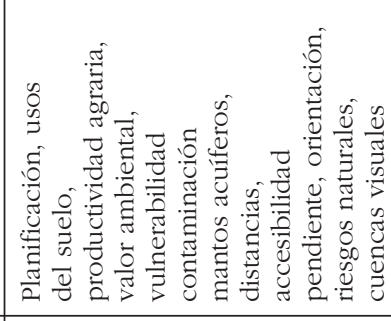 & 1 & 1 & 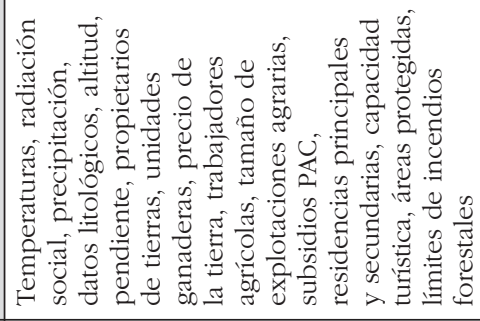 \\
\hline 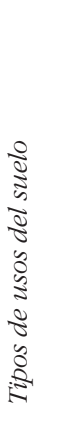 & 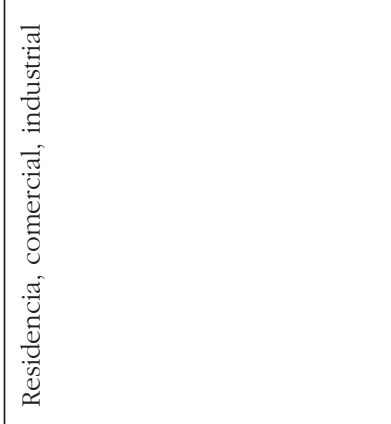 & 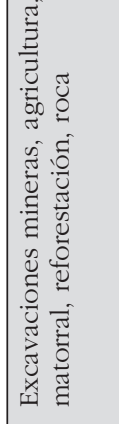 & 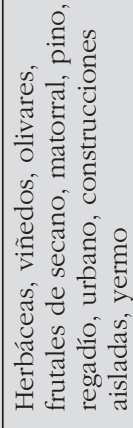 & 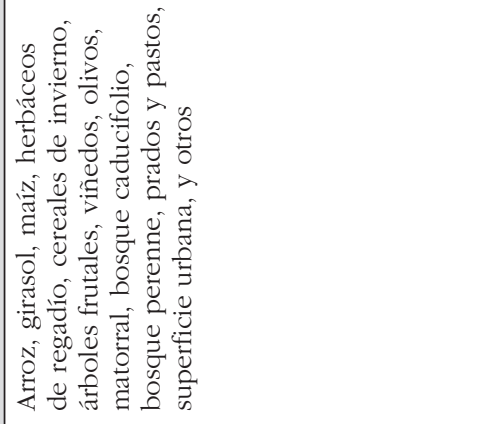 \\
\hline 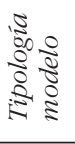 & $\sum_{i=1}^{0}$ & 1 & 1 & 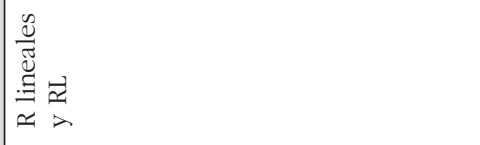 \\
\hline 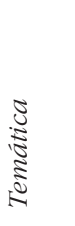 & 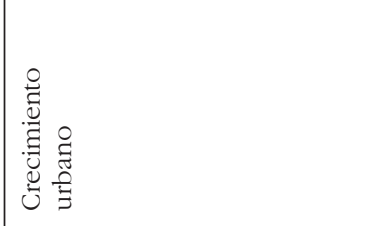 & 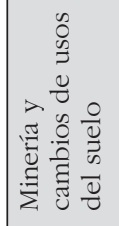 & 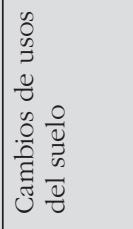 & 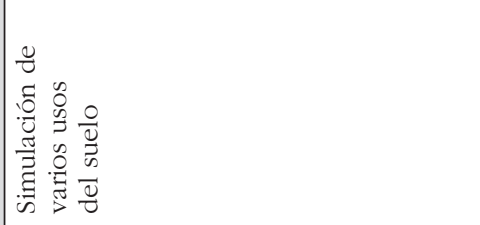 \\
\hline 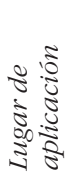 & $\begin{array}{l}\frac{\bar{g}}{\bar{E}} \\
\frac{\pi}{2}\end{array}$ & 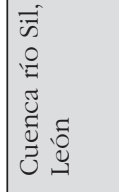 & 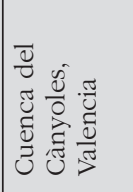 & 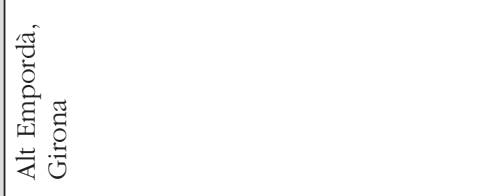 \\
\hline 胥 & 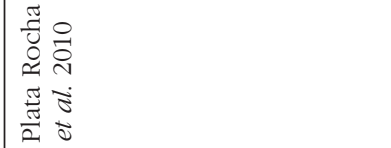 & 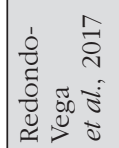 & 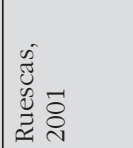 & 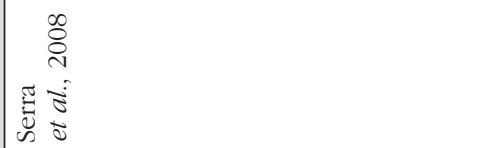 \\
\hline
\end{tabular}

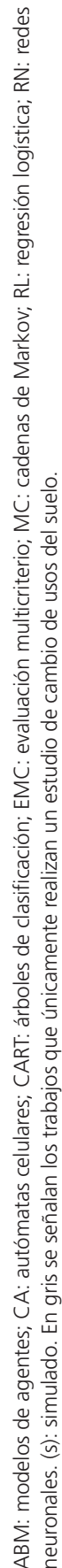




\section{Discusión y conclusiones}

Los estudios de modelos de cambios de usos del suelo y de simulaciones llevados a cabo hasta ahora son muy numerosos. El presente artículo muestra un ejemplo de ello. Para obtener mayor detalle se pueden consultar los trabajos de Briassoulis (2000), donde se recopilan diferentes tipos de modelos, desde econométricos a modelos integrados; Agarwal et al. (2002), los cuales hacen una revisión y evaluación de 19 modelos de cambios de usos del suelo y de simulación de ámbito regional y local, obtenidos del estudio de 136 artículos, incluyendo modelos de Markov, logísticos, econométricos, dinámicos, de CA, etc.; Verburg et al. (2004b) comparan diferentes modelos en relación con el nivel de análisis, la escala espacial y las dinámicas temporales, los factores explicativos y los efectos de vecindad y el nivel de integración; Busch (2006) realiza una selección de 7 estudios y recopila 25 escenarios diferentes a escala global y a escala europea, en relación con paisajes agrarios; y Pontius et al. (2008) y Sohl et al. (2016) realizan una comparación, en cuanto a los datos de entrada y de salida y a la validación de los resultados, de varios estudios que trabajan con diferentes modelos de simulaciones de cambios de usos del suelo; Santé et al. (2010) analizan 33 modelos de simulación de procesos urbanos basados en CA

Parker et al. (2002) indican que los estudios de simulaciones a medio o largo plazo no pueden estar limitados por la observación y la descripción. El desarrollo de modelos causales puede ayudar a comprender la situación actual y reciente, a la vez que proporciona predicciones plausibles. La longitud en el tiempo durante la cual una predicción es válida depende de la persistencia de los fenómenos observados. Hay estudios que sugieren que la mayoría de los cambios en la cubierta terrestre son constantes entre 10 y 15 años; si bien, cambios políticos, institucionales o económicos pueden provocar rápidos cambios en la tasa o en la dirección del cambio de la cubierta.

Busch (2006) señala que los modelos de cambios de usos del suelo y simulaciones se han visto comúnmente desde una perspectiva sectorial, centrados en cambios en la agricultura, las áreas forestales o las áreas urbanas separadamente; sin embargo, cada vez más se están haciendo estudios que integran varios usos del suelo juntos. Además, es necesario elaborar más de un escenario, no solo el escenario tendencial, ya que como comenta ESPON (2007: 7), "la comparación de los resultados de diferentes escenarios es un ejercicio pedagógico cuyo objetivo es la identificación de trayectorias positivas y negativas y de los factores explicativos que las ocasionan para poder configurar un futuro más deseable y prevenir futuros no deseables mediante políticas apropiadas". Por otra parte, se han desarrollado muchos modelos de forma aislada, lo que ha provocado que haya varios modelos que responden a las mismas preguntas y que funcionan de manera similar. Los expertos abogan por la realización de análisis multi-escalares, integrando en la elaboración de los modelos a los diferentes agentes que gobiernan y/o actúan sobre el territorio. 
Los modelos de simulaciones de usos del suelo deben hacer frente a la incertidumbre de éstos. Para Rae et al. (2007) ésta se produce cuando la diferencia entre la información producida y la realidad se desconoce; mientras que el error se produce cuando hay una diferencia medible entre ambas. La incertidumbre y el error en los modelos van más allá del resultado de dicho modelo. Los datos obtenidos de imágenes de satélites o de fotografías aéreas y que funcionan como datos de entrada, contienen ambos aspectos asociados con el tipo de sensor que utilizan o con el procesamiento de imágenes que se lleva a cabo.

\subsection{Problemas o dificultades}

A pesar del número de modelos desarrollados en los últimos tiempos y de su mayor complejidad y sofisticación, éstos siguen reflejando un valor limitado a la hora de simular la complejidad de los factores que se interrelacionan entre sí y que afectan a la evolución territorial (ESPON, 2007).

La definición de las clases de usos del suelo a utilizar tiene un efecto importante en el resultado del modelo, especialmente en modelos que intentan captar el proceso, no sólo el cambio. El uso de una sola clase puede derivar en que el modelo no esté bien calibrado, pero, por otra parte, tener numerosas clases puede reducir su precisión debido a la dificultad de capturar procesos que no son comunes (Conway, 2009). Del mismo modo, cuando se posee una única clase de uso del suelo, donde se estudia la presencia y ausencia de éste, y se desea predecir únicamente dicho cambio, el modelo puede generar menos error que si se estudian varias clases de usos del suelo conjuntamente, ya que hay menos posibilidades de que se convierta a una categoría errónea.

La resolución a la que se trabaja es un elemento determinante. Rindfuss et al., (2004) señalan que la resolución temporal determina el dinamismo que puede ser observado, la resolución espectral afecta a la discriminación del estado del paisaje y su condición, y la resolución radiométrica controla la precisión entre la separación de tipos de cubiertas del suelo y de usos del suelo. Asimismo, se debe tener en cuenta que la resolución espacial y temporal puede no coincidir con los datos biofísicos y socio-económicos con los que se trabajan.

La gran mayoría de los trabajos que se han elaborado sobre cambios de usos del suelo y simulaciones trabajan a nivel de píxel. Un píxel, debido a su forma, nunca podrá representar perfectamente las curvaturas de los polígonos y, tal y como comenta Rae et al. (2007), a la hora de convertir la información de vectorial a ráster, una cantidad considerable de información puede ser perdida.

Los modelos están limitados por la disponibilidad de datos. Agarwal et al. (2002: 28) señalan que hay que tener en cuenta que "cualquier modelo de cambios de usos del 
suelo está, probablemente, limitado por la persona o personas que lo construyen, de acuerdo con sus límites formativos y el financiamiento que poseen" y la posibilidad de que haya o se puedan generar datos consistentes de la zona de estudio.

Existe una gran dificultad para desentrañar los diferentes procesos humanos, sociales, económicos, políticos, medioambientales, biofísicos, etc. que inciden sobre un territorio y que desembocan en cambios de usos del suelo, ya que son numerosos y muy variados los factores que entran en juego.

\subsection{Retos}

Aunque es una tarea difícil, el consenso internacional en la generación y utilización de una cartografía temática a diferentes escalas espaciales, podría conllevar la realización de análisis comparativos entre diferentes zonas, regiones o países, de manera multiescalar. La realización de una cartografía estándar, como CORINE Land Cover (CLC) en Europa, es muy importante pues permite la comparación de las dinámicas de las cubiertas y usos del suelo que se han sucedido en el tiempo y en diferentes ámbitos geográficos, pudiendo hacer comparaciones gracias a la homogeneidad en la leyenda temática. En España, el programa SIOSE permite trabajar a una escala de mayor detalle, siendo su objetivo, al igual que CLC, continuar con la realización de cartografía de cubiertas y usos del suelo en varios momentos en el tiempo para que puedan realizarse estudios temporales y también comparativos. La continuidad de estos trabajos es, por ello, fundamental.

La mayoría de los modelos de usos del suelo que se han llevado a cabo hasta ahora están basados en una única escala espacial. Sin embargo, como proponen Verburg et al. (2004b) se necesita una nueva generación de modelos de usos del suelo que aborden mejor las características multiescalares del sistema de la tierra y que logren un mayor nivel de integración entre diferentes enfoques disciplinarios.

Además, las simulaciones a futuro necesitan ser llevadas a diferentes periodos de tiempo, desde tiempos cortos para el diseño e implementación política, hasta medio y largo plazo para observar los efectos que pueden tener los factores climáticos, sociales, económicos y tecnológicos.

La realización de este tipo de estudios puede enlazarse con otro tipo de trabajos como son los de ecología del paisaje (Turner et al., 2001) o investigaciones que analicen los intercambios y las sinergias en la provisión de servicios del ecosistema o en el cambio climático (Eitelberg et al., 2016).

Por último, es necesario ampliar y profundizar en la participación de actores en la creación de los escenarios y en el plan de trabajo. El compromiso de los agentes es 
fundamental para poder llevar a cabo la creación de posibles futuros deseados. Para ello, se debe integrar no solo a políticos o responsables políticos, sino también a ONGs y empresarios con intereses en los usos del suelo (incluyendo el medio natural). En este sentido, otro reto está en que la implicación de estos profesionales logre materializarse en futuros planeamientos territoriales

\section{Bibliografía}

Abd El-Kawy, O.R., Rød, J.K., Ismail, H.A. y Suliman, A.S., 2011. Land use and land cover change detection in the western Nile delta of Egypt using remote sensing data. Applied Geography 31, pp. 483-494. Doi: 10.1016/j.apgeog.2010.10.012

Alcamo, J., Kok, K., Busch, G. y Priess, J.A., 2006. Searching from the future of land: scenarios from the local to global scale. En: Lambin, E.F. y Geist, H.J. (eds), Land-use and land-cover change. Local processes and Global Impacts, Berlin: Springer, pp. 137-156. Doi: 10.1007/3-540-32202-7

Agarwal, C., Green, G.L., Grove, J.M., Evans, T. y Schweik, C., 2002. A review and assessment of land use change models. Dynamics of space, time and human choice, Gen. Tech. Rep. NE-297. Pennsylvania, Department of Agriculture, Forest Services, Northeastern Research Station, 61 p.

Aguilera, F., Ruiz Matarán, A., Campaña Pérez, R. y Montes Valenzuela, L.M., 2008. Simulating greenhouse growth in urban zoning on the coast of Granada (Spain). En: Paegelow, M. y Camacho Olmedo, M.T. (eds), Modelling Environmental Dynamics. Advances in Geomatic Solutions, Berlin Heidelberg: Springer, pp. 269-295. Doi: 10.1007/978-3-540-68498-5

Aguilera-Benanvente, F., Botequilha-Leitao, A. y Díaz-Varela, E., 2014. Detectiong multi-scale urban growth patterns and processes in the Algarve region (Southern Portugal). Applied Geography, 53, pp. 234-245. Doi: 10.1016/j.apgeog.2014.06.019

Barreira-Gonzalez, P., Gómez-Delgado, M. y Aguilera-Benavente, F., 2015. From raster to vector cellular automata models: a new approach to simulate urban growth with the help of graph theory. Computers, Environment and Urban Systems, 54, pp. 119-131. Doi: 10.1016/j.compenvurbsys.2015.07.004

Bell, E.J., 1974. Markov analysis of land use change - an application of stochastic processes to remotely sensed data. Socio-Economic Planning Sciences, 8, pp. 311-316.

Briassoulis, H., 2000. Analysis of Land Use Change: Theoretical and Modeling Approaches. En: Loveridge, S. (ed), The Web Book of Regional Science. Regional Research Institute, West Virginia University. http://www.rri.wvu.edu/WebBook/Briassoulis/contents.htm

Busch, G., 2006. Future European agricultural landscapes. What can we learn from existing quantitative land use scenarios studies? Agriculture, Ecosystems E Environment, 114, pp. 121-140. Doi: 10.1016/j.agee.2005.11.007

Conway, T.M., 2009. The impact of class resolution in land use change models. Computers, Environment and Urban Systems, 33, pp. 269-277. Doi: 10.1016/j.compenvurbsys.2009.02.001

Deng, Y. y Srinivasan, S., 2016. Urban land use change and regional access: a case study in Beijing, China. Habitat international, 51, pp. 103-113. Doi: 10.1016/j.habitatint.2015.09.007 
Díaz-Pacheco, J y García-Palomares, J.C., 2014. A highly detailed land-use vector map for Madrid region based on photo-interpretation. Journal of Maps, 10(3), pp. 424-433. Doi: 10.1080/ 17445647.2014 .882798

Eitelberg, D.A., van Vliet J., Doelman, J.C., Stehfest, E. y Verburg, P.H., 2016. Demand for biodiversity protection and carbon storage as drivers of global land change scenarios. Global Environmental Change, 40, pp. 101-111. doi: 10.1016/j.gloenvcha.2016.06.014

ESPON, 2007. Scenarios on the territorial future of Europe. Belgium: ESPON 2006 Programme.

Gallardo, M. y Martínez-Vega, J., 2016. Three decades of land-use changes in the region of Madrid and how they relate to territorial planning. European Planning Studies, 24(5), pp. 1016-1033. Doi: 10.1080/09654313.2016.1139059

Gallardo, M y Martínez-Vega, J., 2018. Modelling land-use scenarios in protected areas of an urban region in Spain. En: Camacho Olmedo, M.T., Paegelow, M., Mas, J.F. y Escobar, F. (eds). Geomatic simulations and scenarios for modelling LUCC: A review and comparison of modelling techniques. Springer, pp. 307-328. doi: 10.1007/978-3-319-60801-3

García, A.M., Santé, I., Boullón, M. y Crecente, R., 2012. A comparative analysis of cellular automata models for simulation of small urban areas in Galicia, NW Spain. Computers, Environment and Urban Systems, 36 (4), pp. 291-301. Doi: 10.1016/j.compenvurbsys.2012.01.001

Geoghegan, J., Pritchard, L.J., Ogneva-Himmelberger, Y., Chowdhury, R.R., Sanderson, S. y Turner, B.L.I., 1998. Socializing the pixel and Pixelizing the Social in land-use and land-cover change. En: N.R.C. Staff (ed), People and Pixels: Linking remote sensing and social science. Washington, D.C.: National Academies Press, pp. 51-69.

Gidey, E., Dikinya, O., Sebego, R., Segosebe, E. y Zenebe, A., 2017. Modeling the spatio-temporal dynamics and evolution of land use and land cover (1984-2015) using remote sensing and GIS in Raya, Northern Ethiopia. Modeling Earth Systems and Environments, 3(4), pp. 12851301. doi: 10.1007/s40808-017-0375-Z

Hagen-Zanker, A., 2006. Map comparison methods that simultaneously address overlap and structure. Journal of Geographical Systems, 8, pp. 165-185. Doi: 10.1007/s10109-006-0024-y

Henríquez, C., Azócar, G. y Aguayo, M., 2006. Cambio de uso del suelo y escorrentía superficial: aplicación de un modelo de simulación espacial en Los Angeles, VIII Region del Biobio, Chile. Revista de Geografía Norte Grande, 36, pp. 61-74. Doi: 10.4067/S0718-34022006000200004

Hewitt, R., van Delden, H. y Escobar, F., 2014. Participatory land use modelling, pathways to an integrated approach. Environmental Modelling \& Software, 52, pp. 149-164. Doi: 10.1016/j.envsoft.2013.10.019

Jokar Arsanjani, J., Helbich, M., Kainz, W. y Darvishi Boloorani, A., 2013. Integration of logistic regression, Markov chain and cellular autómata models to simulate urban expansion. International Journal of Applied Earth Observation and Geoinformation, 21, pp. 265-275. Doi: 10.1016/j.jag.2011.12.014

Kok K. y van Delden H., 2013. Linking Narrative Storylines and Quantitative Models to Combat Desertification in the Guadalentín Watershed (Spain). En: Giaoutzi M. y Sapio B. (eds) Recent Developments in Foresight Methodologies. Complex Networks and Dynamic Systems, vol 1. Boston: Springer. doi: 10.1007/978-1-4614-5215-7_13 
Koo, H., Kleemann, J. y Fürst, C., 2018. Land use scenario modeling based on local knowledge for the provision of ecosystem services in Northern Ghana. Land, 7, p. 59. Doi:10.3390/land7020059

$\mathrm{Ku}$, C.A., 2016. Incorporating spatial regression model intro cellular automata for simulating land use change. Applied Geography, 69, pp. 1-9. Doi: 10.1016/j.apgeog.2016.02.005

Lambin, E.F. y Geist, H.J. (eds), 2006. Land-use and land-cover change. Local processes and global impacts. Berlin Heidelberg: Springer. Doi: 10.1007/3-540-32202-7

Lawler, J.J., Lewis, D.J., Nelson, E., Plantinga, A.J., Polasky, S., Withney, J.C., Helmers, D.P., Martinuzzi, S., Pnnington, D. y Radeloff, V.C., 2014. Projected land-use change impacts on ecosystems services in the United States. PNAS, 11 (20), pp. 7492-7497. Doi: 10.1073/pnas.1405557111

Li, X., y Yeh, A.G., 2002. Neural-network-based cellular automata for simulating multiple land use changes using GIS. International Journal of Geographical Information Science, 16, pp. 323343. Doi: 10.1080/13658810210137004

Lima, L., Zelaya, K., Laterra, P., Massone, H. y Maceira, N., 2011. A dynamic simulation model of land cover in the Dulce Creek Basin, Argentina. Procedia Environmental Sciences, 7, pp. 194-199. Doi: 10.1016/j.proenv.2011.07.034

Malek, Z, Verburg, P.H. y Geijzendorffer, I.R., 2018. Global change effects on land management in the Mediterranean region. Global Environmental Change, 50, pp. 238-254. doi: 10.1016/ j.gloenvcha.2018.04.007

Martínez-Fernández, J., Ruiz-Benito. P. y Zavala, M.A., 2015. Recent land cover changes in Spain across biogeographical regions and protection levels: Implications for conservation policies. Land Use Policy, 44, pp. 62-75. Doi: 10.1016/j.landusepol.2014.11.021

Martinuzzi, S., Radeloff, V.C., Joppa, L.N., Hamilton, C.M., Helmers, D.P., Plantinga, D.P. y Lewis, D.J., 2015. Scenarios of future land use change around United States' protected areas. Biological Conservation, 184, pp. 446-455. Doi: 10.1016/j.biocon.2015.02.015

McDonald, R.I. y Urban, D.L., 2006. Spatially varying rules of landscape change: lessons from a case study. Landscape and Urban Planning, 74, pp. 7-20. Doi: 10.1016/j.landurbplan.2004.08.005

Moore, I.D., Lee, B.G. y Davey, S.M., 1991. A new method for predicting vegetation distributions using decision tree analysis in a geographic information system. Environmental Management, 15, pp. 59-71. Doi: 10.1007/BF02393838

Otto, R., Krüsi, B.O. y Kienast, F., 2007. Degradation of an arid coastal landscape in relation to land use changes in Southern Tenerife (Canary Islands). Journal of Arid Environments, 70, pp. 527-539. 10.1016/j.jaridenv.2007.02.001

Overmars, K.P., Verburg, P.H. y Veldkamp, T., 2007. Comparison of a deductive and an inductive approach to specify land suitability in a spatially explicit land use model. Land Use Policy, 24, pp. 584-599. Doi: 10.1016/j.landusepol.2005.09.008

Paegelow, M., Camacho, M.T. y Menor, J., 2003. Cadenas de Markov, evaluación multicriterio y evaluación multiobjetivo para la modelización prospectiva del paisaje. Geofocus, 3, pp. 24-44.

Parker, D.C., Berger T. y Manson, S.M., 2002. Agent-based models of Land use and Land-cover change. LULC Report Series No.6. Louvain-la-Nueve, LUCC International Project Office. 
Peña, J., Bonet, A., Bellot, J., Sánchez, J.R., Eisenhulth, D., Hallett, S. y Aledo, A., 2007. Driving forces of land-use change in a cultural landscape of Spain. En: Koomen, K., Stillwell, J., Bakema, A. y Scholten, H.J. (eds) Modelling land-use change. Progress and applications. Springer Netherlands, pp. 97-115. Doi: 10.1007/978-1-4020-5648-2

Plata Rocha, W., Gómez Delgado, M. y Bosque Sendra, J., 2010. Desarrollo de modelos de crecimiento urbano óptimo para la Comunidad de Madrid aplicando métodos de evaluación multicriterio y Sistemas de Información Geográfica. Geofocus, 10, pp. 103-134.

Pontius Jr, R.G., Huffaker, D. y Denman, K., 2004. Useful techniques of validation for spatially explicit land-change models. Ecological Modelling, 179, pp. 445-461. Doi: 10.1016/j.ecolmodel.2004.05.010

Pontius Jr, R.G., Boersma, W. Castella, J.C., Clarke, K., De Nijs, T.C.M., Dietzel, C., Duan, Z., Fotsing, E., Goldstein, N., Kok, K., Koomen, E., Lippitt, C.D., McConnell, W., Sood, A.M., Pijanowski, B., Pithadia, S., Sweeney, S., Trung, T.N. Veldkamp, A.T. y Verburg, P.H., 2008. Comparing the input, output, and validation maps for several models of land change. Annals of Regional Science, 42, pp. 11-37. Doi: 10.1007/s00168-007-0138-2

Rae, C., Rothley, K. y Dragicevic, S., 2007. Implications of error and uncertainty for an environmental planning scenario: A sensitivity analysis of GIS-based variables in a reserve design exercise. Landscape and Urban Planning, 79, pp. 210-217. Doi: 10.1016/j.landurbplan.2006.01.001

Redondo-Vega, J.M., Gómez-Villar, A., Santos-González, J., González-Gutiérrez, R.B., y ÁlvarezMartínez, J., 2017. Changes in land use due to mining in the north-western mountains of Spain during the previous 50 years. Catena, 149, pp. 844-856. Doi: 10.1016/j.catena.2016.03.017

Reid, R.S., Tomich, T.P., Xu, J.C., Geist, H.J. y Mather, A.S., 2006. Linking land-change science and policy: current lessons and future integration. En: Lambin, E.F. y Geis, H.J. (eds) Land-use and land-cover change. Local processes and Global Impacts, Berlin: Springer, pp. 157-172. Doi: 10.1007/3-540-32202-7

Rindfuss, R.R., Walsh, S.J., Turner, B.L.I., Fox, J. y Mishra, V., 2004. Developing a science of land change: challenges and methodological issues. PNAS, 101, pp. 13976-13981. Doi: 10.1073/ pnas. 0401545101

Romano, B., Zullo, F., Fiorini, L., Marucci, A. y Ciabò, S., 2017. Land transformation of Italy due to half a century of urbanization. Land use policy, 67, pp. 387-400. Doi: 10.1016/j.landusepol.2017.06.006

Rounsevell, M.D.A., Pedroli, B., Erb, K.H., Gramberger, M., Busck, A.G., Haberl, H., Kristensen, S., Kuemmerle, T., Lavorel, S., Lindner, M., Lotze- Campen, H., Metzger, M.J., Murray-Rust, D., Popp, A., Pérez-Soba, M., Reenberg, A. Vadineanu, A., Verburg, P.H. y Wolfslehner, B., 2012. Challenges for land system science. Land Use Policy, 29, pp. 899-910. Doi: 10.1016/j.landusepol.2012.01.007

Ruescas, A.B., 2001. Análisis de la dinámica espacio-temporal de los usos del suelo mediante SIG: la cabecera de la cuenca de Canyoles. Cuadernos de Geografía, 69, pp. 251-230.

Sangermano, F., Toledano, J., y Eastman, J.R., 2012. Land cover change in the Bolivian Amazon and its implications for REDD+ and endemic biodiversity. Landscape Ecology, 27, pp. 871-584. Doi: 10.1007/s10980-012-9710-y 
Santé, I., García, A.M., Miranda, D. y Crecente, R., 2010. Cellular automata models for the simulation of real-word urban processes: A review and analysis. Landscape and Urban Planning, 96, pp. 108-122. Doi: 10.1016/j.landurbplan.2010.03.001

Serra, P., Pons, X. y Sauri, D., 2008. Land-cover and land-use change in a Mediterranean landscape: a spatial analysis of driving forces integrating biophysical and human factors. Applied Geography, 20, pp. 189-209. Doi: 10.1016/j.apgeog.2008.02.001

Sohl, T., Wimberly, M.C., Radeloff, V.C., Theobald, D.M. y Sleeter, B.M., 2016. Divergent projections of future land use in the United States arising from different models and scenarios. Ecological Modelling, 337, pp. 281-297. Doi: 10.1016/j.ecolmodel.2016.07.016

Titeux, N., Henle, K., Mihoub. J.B., Regos, A., Geizendorffer, I.R., Cramer, W., Verburg, P.H. y Brotons, L., 2017. Global scenarios for biodiversity need to better integrate climate and land use change. Diversity and Distributions, 23, pp. 1231-1234. doi: 10.1111/ddi.12624

Tobler, W., 1970. A computer movie simulating urban growth in the Detroit region. Economic Geography, 46 (2), pp. 234-240.

Turner, M.G., Gardner, R.H. y O'Neill, R.V., 2001. Landscape ecology in theory and practice. Pattern and process. New York: Springer-Verlag.

Vafeidis, A.T., Kokoulas, S., Gatsis, I. y Gkoltsiou, K., 2007. Forecasting land use changes with the use of neuronal networks and GIS. 2007 IEEE International Geoscience and Remote Sensing Symposium, Barcelona, pp. 5068-5071, doi: 10.1109/IGARSS.2007.4424001

Valbuena, D., Verburg, P.H., y Bregt, A.K., 2008. A method to define a typology for agent-based analysis in regional land-use research. Agriculture, Ecosystems \& Environment, 128, pp. 27-36. Doi: 10.1016/j.agee.2008.04.015

Veldkamp, A., y Fresco, L.O., 1997. Exploring land use scenarios, an alternative approach based on actual land use. Agricultural Systems, 55, pp. 1-17. Doi: 10.1016/S0308-521X(95)00079-K

Veldkamp, A., y Lambin, E.F., 2001. Predicting land-use change. Agriculture, Ecosystems and Environment, 85, pp. 1-6. Doi. 10.1016/S0167-8809(01)00199-2

Verburg, P.H., Ritsema van Eck, J., De Nijs, T.C.M., Dijst, M.J. y Schot, P., 2004a. Determinants of land-use change patterns in the Netherlands. Environment and Planning B: Planning and Design, 31, pp. 125-150. Doi: 10.1068/b307

Verburg, P.H., Schot, P.P, Dijst, M.J. y Veldkamp, A., 2004b. Land use change modelling: current practice and research priorities. GeoJournal, 61, pp. 309-324. Doi: 10.1007/s10708-004-4946-y

Verburg, P.H., Kok, K., Pontius Jr, R.G. y Veldkamp, A., 2006a. Modeling land-use and land-cover change. En: Lambin, E.F. y Geist, H.J. (eds). Land-use and land-cover change. Local processes and Global Impacts. Berlin: Springer, pp. 117-135. Doi: 10.1007/3-540-32202-7

Verburg, P.H., Rounsevell, M.D.A y Veldkamp, A., 2006b. Scenario-based studies of future land use in Europe. Agriculture, Ecosystems E Environment, 114, pp. 1-6. Doi: 10.1016/j.agee. 2005. 11.023

Verburg, P.H., Schulp, C.J.E., Witte, N. y Veldkamp, A., 2006c. Downscaling of land use change scenarios to assess the dynamics of European landscapes. Agriculture, Ecosystems \& Environment, 114, pp. 39-56. Doi: 10.1016/j.agee.2005.11.024 
Wassenaar, T., Gerber, P., Verburg, P.H., Rosales, M., Ibrahim, M. y Steinfeld, H., 2007. Projecting land use changes in the Neotropics: The geography of pasture expansion into forest. Global Environmental Change, 17, pp. 86-104. Doi: 10.1016/j.gloenvcha.2006.03.007

Xu, C. Haase, D. y Pauleit, S., 2018. The impact of different urban dynamics on green space availability: a multiple scenario modeling approach for the region of Munich, Germany. Ecological Indicators, 93, pp. 1-12. Doi: g/10.1016/j.ecolind.2018.04.058 